\section{Prevalência e características associadas à síndrome metabólica em nipo-brasileiros com e sem doença periodontal}

\author{
Prevalence and characteristics associated with \\ metabolic syndrome in Japanese-Brazilians with \\ and without periodontal disease
}

Pollyanna Kássia de Oliveira Borges 1 Suely Godoy Agostinho Gimeno 1

Nilce Emy Tomita 2

Sandra Roberta Ferreira 1

Japanese-Brazilians Diabetes Study Group ${ }^{3}$
${ }^{1}$ Escola Paulista de Medicina, Universidade Federal de São Paulo, São Paulo, Brasil. 2 Faculdade de Odontologia de Bauru, Universidade de São Paulo, Bauru, Brasil. 3 Outros membros listados ao final do artigo.

Correspondência P. K. O. Borges Escola Paulista de Medicina Universidade Federal de São Paulo.

Rua Onofre Pereira de Matos 1070, apto. 04, Dourados, MS 79802-010, Brasil. pollyannakassia@hotmail.com

\section{Abstract}

This cross-sectional study focused on the relationship between periodontal disease and metabolic syndrome. Data were analyzed from 1,315 Japanese-Brazilians ranging from 30 to 92 years of age, submitted to physical, laboratory, and dental exams. Means and percentages were used in the data description and logistic regression pattern in the analysis of associations among variables. 484 (36.8\%) of the 1,315 were edentulous, 215 (16.4\%) enjoyed periodontal health, 513 (39\%) had gingivitis, 85 (6.5\%), showed initial or moderate periodontitis, and 18 (1.4\%) suffered from chronic periodontits. Prevalence of metabolic syndrome was 54.3\%, higher among individuals with periodontitis than in the healthy (51.5\% vs. 48.8\%), but this association was not statistically significant. Individuals with metabolic syndrome showed a worse metabolic and anthropometric profile.

Metabolic Syndrome X; Diabetes Mellitus; Dyslipidemias; Periodontal Diseases; Obesity

\section{Introdução}

A síndrome metabólica é o nome proposto pela Organização Mundial da Saúde (OMS) 1 e pelo National Cholesterol Education Program (NCEP) 2 para designar um conjunto de alterações metabólicas (dislipidemia, hipertensão arterial, intolerância à glicose, obesidade central e resistência à insulina) que comumente se manifestam juntas e são fatores de risco para a doença coronariana 3,4,5. Cada componente da síndrome metabólica, por si só, aumenta o risco de doença cardiovascular, porém, quando combinado, ele se torna muito mais intenso 6 . A resistência à insulina pode ser um fator etiopatogênico comum aos elementos da síndrome metabólica, embora essas conexões não estejam completamente entendidas 1,5 .

Órgãos internacionais tais como a OMS e o NCEP sugeriram distintas classificações para a síndrome metabólica, todas elas baseadas em combinações de seus componentes. Segundo os pesquisadores que integram o NCEP, um indivíduo deve ser considerado como com síndrome metabólica quando tiver três ou mais alterações metabólicas associadas (obesidade abdominal, hipertrigliciridemia, HDL-colesterol reduzido, hipertensão arterial e glicemia de jejum alterada) 2. Grundy et al. 3 afirmam que esse critério é útil para identificar pacientes com risco aumentado para a doença cardiovascular. 
Rosenbaum et al. 7, em estudo comparativo de diferentes critérios para a identificação de indivíduos com síndrome metabólica, sugeriram que, para a comunidade nipo-brasileira, os padrões de circunferência de cintura propostos pelo NCEP fossem ajustados para o perfil corporal desta população.

As diferentes classificações da síndrome metabólica 1,2,8 têm proporcionado distintos valores da prevalência da doença em todo o mundo; estima-se que cerca de 20 a $25 \%$ da população mundial tenham essa síndrome 9 .

Estudos norte-americanos mostram que 20$30 \%$ da população adulta têm síndrome metabólica 10,11. No Japão, segundo Shimamoto 12 e Sone et al. 13 , mais de $20 \%$ da população geral apresentam resistência à insulina e, na população adulta com diabetes mellitus do tipo 2 , a prevalência da síndrome metabólica varia de 38 a 53\%, a depender do sexo e do critério utilizado para definir a síndrome metabólica.

Dados representativos da prevalência da síndrome metabólica na população brasileira praticamente não existem. O que pode ser encontrado são estudos realizados em populações com características específicas tais como a população de migrantes japoneses. Pelos critérios do NCEP 2, a população nipo-brasileira apresentou elevada prevalência da síndrome metabólica (57\%) e de seus componentes - intolerância à glicose $(69,5 \%)$, dislipidemia (66\% com hipertrigliceridemia e $31,2 \%$ com HDL colesterol diminuído), obesidade central $(42,4 \%)$, hipertensão arterial (56,8\%) 14,15 .

A etiologia da síndrome metabólica é desconhecida, mas provavelmente ocorre a partir de uma interação complexa entre fatores genéticos, metabólicos e ambientais. Resultados de diversos estudos sugerem que o estado pró-inflamatório pode contribuir para o desenvolvimento dessa síndrome 16 .

Processos inflamatórios crônicos relacionados à deposição visceral de gordura estimulam uma ampla ativação do sistema imune que, por sua vez, está envolvida na patogênese da resistência à insulina, do diabetes mellitus do tipo 2, das dislipidemias e da aterosclerose 17,18,19.

Gengivites e periodontites são as qualificações mais comuns de doenças periodontais. Essas doenças são reflexos de infecções bacterianas crônicas e, assim como em outras infecções, a interação hospedeiro-bactéria determina a natureza e a extensão da doença 20,21,22. As gengivites são doenças que causam vermelhidão das gengivas, alterações de contorno, sangramento à sondagem, edema e aumento do fluído gengival; porém os achados clínicos e radiográficos não denotam perda de inserção periodontal e osso alveolar. Já nas periodontites, a inflamação gengival se estende para o sistema de suporte do dente (ligamento periodontal, cemento radicular e osso alveolar) e as perdas de suporte ósseo e de tecido conjuntivo são achados clínicos característicos dessas doenças.

Uma gengivite não evolui necessariamente para uma periodontite e um indivíduo pode conviver com esta doença por toda a vida e não apresentar nenhuma perda dental. Os achados histopatológicos iniciais das periodontites são muitas vezes similares à inflamação das gengivas; porém, apesar dessa semelhança, existem diferenças significativas quanto aos agentes etiológicos e progressão da doença que as tornam entidades diferentes, embora os sinais clínicos iniciais serem parecidos 20,21,23.

As periodontites são doenças infecciosas causadas predominantemente por bactérias anaeróbias gram-negativas que liberam endotoxinas ativadoras de citocinas pró-inflamatórias (IL1 $\beta$, TNF- $\alpha, \mathrm{PGE}_{2}$, e outras), enquanto que as gengivites crônicas são causadas por cocos e bacilos aeróbios gram-positivos. A capacidade invasiva e de toxicidade das bactérias causadoras das periodontites é superior à das gengivites. De igual modo, nas periodontites, o epitélio ulcerado das bolsas periodontais serve como meio de entrada para as bactérias e seus produtos na corrente circulatória, além disso, pela maior capacidade de virulência, as periodontites ativam a resposta inflamatória local em proporções muito superiores que nas gengivites. Portanto, poderiam ser lançados na corrente circulatória produtos da inflamação periodontal relacionados com a etiologia da síndrome metabólica. E, de outro modo, as periodontites poderiam estimular os hepatócitos a produzir citocinas em quantidade maiores 20,21,23,24.

A diversidade nos métodos e índices para classificar, descrever e medir as doenças periodontais limita a interpretação e análises dos dados disponíveis nos estudos populacionais ao redor do mundo. Estima-se que mais da metade da população adulta americana tenha sangramento gengival. Além disso, as melhores condições de higiene oral, os programas de controle do tabagismo e outros de promoção de saúde poderão antecipar um decréscimo na prevalência e gravidade da perda de inserção periodontal nas populações jovens, juntamente com um aumento das doenças periodontais em grupos de idade avançada $25,26,27$.

Tradicionalmente, espera-se que as complicações sistêmicas modulem a resposta inflamatória no periodonto. Entretanto, a partir da década de 90, seguindo a recomendação da American Academy of Periodontology 23, diversos estudos 
foram desenvolvidos para avaliar a relação entre a doença periodontal e as disfunções sistêmicas. Agora, no sentido inverso, o papel das doenças periodontais sobre as alterações sistêmicas estaria relacionada à alta produção de citocinas próinflamatórias, lançadas na corrente sanguínea, durante os períodos de doença periodontal ativa, que, por sua vez, estariam implicadas no aparecimento ou agravamento de diversas condições metabólicas sistêmicas 24,28.

Nishimura \& Murayama 29 comentam que os níveis de proteína C-reativa estão elevados nas periodontites, indicando que a infecção periodontal pode estimular os hepatócitos a sintetizar proteínas inflamatórias. Além disso, os autores articulam que em casos de periodontite avançada, o TNF- $\alpha$ poderia ser secretado tanto pelos tecidos periodontais quanto pelo fígado, em resposta aos lipopolissacarídeos bacterianos das doenças periodontais, contribuindo para o estado de resistência insulínica. Destaca-se que a terapia periodontal pode auxiliar na diminuição dos mediadores inflamatórios circulantes e, conseqüentemente, melhorar a resistência à insulina dos indivíduos 29,30.

A população de origem japonesa (nikkei) se constitui em interessante modelo para o estudo da síndrome metabólica (e de suas complicações), bem como seu relacionamento com as doenças periodontais. Originariamente, esta população apresentava baixa morbidade por diabetes mellitus e por doenças cardiovasculares. Após sofrer importantes mudanças sócio-culturais, em curto período de tempo, passou a apresentar alto risco para esse grupo de doenças 15,31. Além disso, estudos prévios mostraram elevada freqüência de doenças periodontais (45\%) e edentulismo (40\%) entre nipo-brasileiros com intolerância à glicose 32,33 , sugerindo uma possível relação entre doenças periodontais e síndrome metabólica. Assim, julgou-se oportuno investigar, na comunidade nipo-brasileira, a existência de associação entre as presenças de doença periodontal e de síndrome metabólica, controlando-se o efeito de possíveis fatores de confusão.

\section{Metodologia}

Essa investigação foi baseada em dados de um estudo epidemiológico do tipo transversal. Foram utilizadas informações referentes à segunda fase do estudo realizado pelo Japanese-Brazilian Diabetes Study Group (JBDSG) com essa comunidade. Detalhes sobre os procedimentos utilizados foram fornecidos em publicações anteriores $15,32,33$.
A população foi identificada mediante levantamento demográfico realizado em 1997 na comunidade nipo-brasileira residente na cidade de Bauru, São Paulo. Nessa ocasião, foram identificados 1.751 indivíduos de primeira e de segunda geração, com idade $\geq 30$ anos. Todos foram convidados a participar do estudo e 1.330 (76\%) responderam positivamente ao convite. Entre aqueles que não participaram do estudo, observou-se maior proporção de indivíduos do sexo masculino e com idade $\leq 60$ anos quando comparados aos participantes. Das 421 (24\%) não participações, 57 se deram por mudança de cidade ou até de país durante a coleta dos dados e 94 foram por óbitos durante o período da realização do estudo. Logo, do total de indivíduos capazes de participar do estudo $(\mathrm{n}=1.600), 270$ (17\%) se recusaram.

Entrevistadores treinados realizaram visitas domiciliares e aplicaram questionários padronizados. Por meio destes, foram obtidas informações sobre sexo, idade, geração, tabagismo, escolaridade, além de atividade física e hábitos alimentares. Ao final da entrevista, os indivíduos foram convidados a comparecer ao Hospital de Reabilitação de Anomalias Craniofaciais da Universidade de São Paulo, em Bauru, para a realização de exames clínicos, laboratoriais e odontológicos.

O peso corporal foi obtido por meio de balança digital da marca Filizola (São Paulo, Brasil), com capacidade de $200 \mathrm{~kg}$ e precisão de $100 \mathrm{~g}$, estando o indivíduo com o mínimo de roupa possível e sem sapatos. A estatura foi medida de forma direta, utilizando-se estadiômetro acoplado à parede, com o sujeito ereto, descalço ou somente com meias, com os calcanhares unidos na barra de medida, em superfície lisa, plana e rígida, com os braços pendentes ao longo do corpo. $\mathrm{O}$ índice de massa corporal (IMC) foi obtido como a razão entre o peso $(\mathrm{kg})$ e o quadrado da altura $\left(\mathrm{m}^{2}\right)$. A circunferência da cintura foi medida com fita métrica inextensível, na altura da cicatriz umbilical. Esta medida foi realizada com o indivíduo ereto, abdômen relaxado, braços ao lado do corpo e os pés juntos. A presença de obesidade abdominal foi assinalada por valores de circunferência da cintura $\geq 80$ e $90 \mathrm{~cm}$ para o sexo feminino e masculino, respectivamente 7,34 .

As medidas de pressão arterial sistólica e diastólica foram realizadas por médicos treinados, utilizando-se aparelhos digitais automáticos com adequação do manguito à circunferência braquial, modelo HEM-712C do fabricante Omron (Tóquio, Japão), após dez minutos de repouso na posição sentada. Três medidas foram realizadas e o valor final considerado foi aquele que representou a média aritmética das duas úl- 
timas e foi expresso em milímetros de mercúrio (mmHg). Foram considerados hipertensos aqueles indivíduos que apresentaram valor de pressão arterial sistólica $\geq 130 \mathrm{mmHg}$ e pressão arterial diastólica $\geq 85 \mathrm{mmHg}$ ou em uso regular de medicação anti-hipertensiva ${ }^{2}$.

Amostras de sangue foram coletadas após jejum de pelo menos dez horas. Essa medida foi utilizada para várias determinações incluindo perfil lipídico, de glicose e de insulina. Os lípides séricos foram medidos utilizando métodos enzimáticos. A presença de dislipidemia foi definida pela presença de triglicérides $\geq 150 \mathrm{mg} / \mathrm{dl}$ ou de $\mathrm{HDL}<40 \mathrm{mg} / \mathrm{dl}$ no sexo masculino e $<50 \mathrm{mg} / \mathrm{dl}$ no sexo feminino 2 . A glicemia plasmática foi determinada pelo método da glicose-oxidase. Foram considerados com intolerância à glicose aqueles com glicemia de jejum acima de $110 \mathrm{mg} / \mathrm{dl} 2$.

Os níveis séricos de insulina foram dosados por método imunofluorimétrico (AutoDelphia). A avaliação de resistência insulínica foi realizada por meio do índice HOMA-resistência à insulina segundo Matthews et al. 35. Utilizou-se ensaio imunométrico de quimioluminescência para determinação dos valores da PCR (variação normal: $0,05-0,11 \mathrm{mg} / \mathrm{dl})$. A leptina foi dosada por radioimunoensaio (Linco Research Inc., St. Charles, Estados Unidos). O soro foi estocado em temperatura de $-20^{\circ} \mathrm{C}$ para a determinação dos hormônios.

O ensaio para a determinação dos níveis plasmáticos totais de homocisteína baseou-se em metodologia descrita por Pfeiffer et al. 36, para aplicação em cromatografia líquida de alto desempenho (HPLC - high performance liquid chromatography) com detecção fluorimétrica e eluição isocrática; as análises foram feitas em aparelho que consistiu em um HPLC, marca Shimadzu (Kyoto, Japão), com injetor automático de amostras SIL-10Advp e um detector de fluorescência RF-10AXL.

Foram empregadas as recomendações do NCEP do ano de 20012 para a classificação dos indivíduos quanto à síndrome metabólica, com as modificações para a obesidade abdominal sugeridas por Rosenbaum et al. ${ }^{7}$ para a população nipo-brasileira. Assim, foram considerados como com síndrome metabólica, os nipo-brasileiros com pelo menos três das seguintes alterações metabólicas: obesidade abdominal, dislipidemia, intolerância à glicose ou hipertensão arterial.

Profissionais treinados realizaram os exames odontológicos em local apropriado, com iluminação indireta, em macas, com o sujeito deitado e a cabeça apoiada. Na avaliação das condições de saúde bucal foram empregados o índice periodontal comunitário (CPI) e o índice de perda de inserção periodontal (PIP) 37,38. Em ambos os casos, a cavidade oral foi dividida em seis sextantes, para que os dentes índices fossem examinados (dentes 17, 16, 11, 26, 27, 37, 36, 31, 46 e 47).

No exame do CPI, cada dente índice foi avaliado com a sonda periodontal CPI em pelo menos seis diferentes regiões e a pior situação encontrada foi considerada como representativa de cada sextante (higidez, sangramento provocado à sondagem, presença de cálculo, bolsas de 4$5 \mathrm{~mm}$, bolsas periodontais de $6 \mathrm{~mm}$ ou mais ou edentulismo no sextante). Para os sextantes onde não havia dente índice, todos os outros dentes do sextante (exceto a face distal do terceiro molar) foram examinados. O sextante foi excluído (não examinado) quando havia apenas um dente presente, ou quando os dentes tinham ou indicação de extração (lesão de furca ou mobilidade) ou presença de implante dentário 32,33 .

Nesse estudo, utilizou-se o índice CPI para caracterizar a presença de doença periodontal. Os indivíduos foram classificados como tendo higidez periodontal, ou gengivite (presença de sangramento a sondagem ou cálculo), ou periodontite inicial a moderada (bolsas periodontais de $4-5 \mathrm{~mm}$ ), ou periodontite avançada (bolsas periodontais com $6 \mathrm{~mm}$ ou + ), ou edentulismo total, considerando-se a pior condição encontrada em qualquer dos sextantes. Assim, mesmo que o paciente tivesse cinco sextantes hígidos e apenas um sextante com periodontite, ele foi classificado como com periodontite ${ }^{39}$. Por ser mais plausível e aceito pela comunidade científica 28,29 , foram excluídos da análise da possível associação entre síndrome metabólica e doenças periodontais os indivíduos com gengivite ou com edentulismo. Assim, foram analisados somente os indivíduos com periodonto hígido e os com periodontite $(n=318)$.

O índice PIP foi utilizado na avaliação da inserção periodontal. A referência para esse exame foi a junção cemento-esmalte - quando esta não estava visível e o índice CPI indicava higidez, sangramento à sondagem ou presença de cálculo, a perda de inserção foi considerada nula. Considerou-se como com perda de inserção aqueles indivíduos com junção cemento-esmalte visível ou não, mas com índice CPI indicativo de bolsas periodontais acima de quatro milímetros. De acordo com a recomendação da American Academy of Periodontology 39, os nipo-brasileiros foram classificados - segundo a pior condição encontrada em qualquer dos sextantes - como com higidez ou com perda de inserção inicial a moderada (perda de inserção de 4-5mm) ou com perda de inserção avançada (perda de inserção de $6-11 \mathrm{~mm}$ ) ou com indicação para exodontia (perda de inserção acima de $12 \mathrm{~mm}$ ) ou edentulismo total. Neste estudo, o índice PIP foi utiliza- 
do apenas para descrever a possível experiência anterior de doenças periodontais.

\section{Análise dos dados}

As presenças de síndrome metabólica e de periodontites foram consideradas, respectivamente, como variáveis dependente e independente de principal interesse. Foram utilizadas, como variáveis de controle (ou de confusão), sexo, idade, IMC, escolaridade (anos de estudo), níveis séricos de creatinina, ácido úrico, leptina, homocisteina e proteína C-reativa.

De forma a descrever os dados, utilizaram-se os valores percentuais, médios e os desvios padrão das variáveis antropométricas, metabólicas e biológicas dos indivíduos agrupados conforme a condição periodontal dada pelo CPI. Em análise bruta, a existência de associações entre as variáveis foi verificada mediante o emprego das estatísticas qui-quadrado e odds ratio (OR).

O modelo de regressão logística foi utilizado para obter, na presença da síndrome metabólica, o valor do OR para as periodontites, ajustado aos possíveis fatores de confusão. Todas as variáveis independentes que, em análise bruta apresentaram valor de $\mathrm{p}<0,20$ compuseram o modelo inicial; uma a uma, foram eliminadas aquelas cuja saída não implicasse em alteração $\geq 20 \%$ no valor dos odds ratios da variável de exposição de principal interesse (doenças periodontais) 40.

Utilizou-se na análise dos dados o programa Stata 8.0 (Stata Corporation, College Station, Estados Unidos).

O estudo foi aprovado pelo Comitê de Ética em Pesquisa da Escola Paulista de Medicina, Universidade Federal de São Paulo e todos os participantes formalizaram sua concordância mediante assinatura em termo de consentimento livre e esclarecido.

Essa pesquisa foi elaborada de acordo com os princípios éticos contidos na Declaração de Helsinki, da World Medical Association 41.

\section{Resultados}

A idade média dos 1.315 nipo-brasileiros submetidos ao exame odontológico foi de 57 anos (desvio padrão $=12,5$ anos); destes $53,8 \%$ eram do sexo feminino e $80,6 \%$ de segunda geração.

Do total de pacientes examinados, 215 $(16,4 \%)$ apresentaram higidez periodontal, 484 $(36,8 \%)$ edentulismo total, 513 (39\%) gengivites, $85(6,5 \%)$ periodontite inicial ou moderada e 18 $(1,47 \%)$, periodontite avançada. Independente das condições de saúde bucal, a prevalência observada de síndrome metabólica foi de 54,3\%
(Tabela 1). Os valores de prevalência da síndrome metabólica entre os indivíduos sadios, com gengivite, periodontite inicial a moderada, periodontite avançada e edentulismo, foram, respectivamente, $48,8 \%, 50,5 \%, 48,2 \%, 66,7 \%, 61,4 \%$ (Tabela 1). Porém, quando foi considerada apenas a presença de periodontite, essas prevalências foram: $51,5 \%$ e $48,8 \%$, entre os que apresentavam periodontite e entre os sadios, respectivamente. Por meio do exame PIP, constatou-se que 15,6\% dos sujeitos tinham inserção periodontal íntegra, $22,8 \%$ perda de inserção de inicial a moderada, $22,2 \%$ perda de inserção avançada e 2,6\% indicação para exodontia (dados não apresentados).

\begin{tabular}{|c|c|c|}
\hline \multicolumn{3}{|c|}{$\begin{array}{l}\text { Prevalência da síndrome metabólica em nipo-brasileiros segundo as diferentes } \\
\text { condições de saúde bucal obtidas pelo índice periodontal comunitário. }\end{array}$} \\
\hline \multirow[t]{2}{*}{ Condição de saúde bucal } & \multicolumn{2}{|c|}{ Síndrome metabólica } \\
\hline & $\operatorname{Sim}(\%)$ & Não (\%) \\
\hline Higidez & 48,8 & 51,2 \\
\hline Gengivite & 50,5 & 49,5 \\
\hline Periodontite inicial a moderada & 48,2 & 51,8 \\
\hline Periodontite avançada & 66,7 & 33,3 \\
\hline Edentulismo & 61,4 & 38,6 \\
\hline Total & 54,3 & 45,7 \\
\hline
\end{tabular}

São descritos, na Tabela 2 os valores das médias e dos desvios padrão de variáveis quantitativas dos nipo-brasileiros, segundo a condição de saúde bucal definida pelo índice CPI. Foram observados valores médios de idade superior nos sujeitos com periodontite avançada ou com edentulismo (58,7 e 65,5 anos) quando comparados aos outros grupos do CPI. Os níveis médios de homocisteína e PCR foram maiores naqueles com periodontite inicial/moderada, periodontite avançada e edentulismo quando comparados aos com gengivite ou sadios; valores médios de ácido úrico e creatinina também se apresentaram discretamente superiores nos sujeitos com gengivite, periodontite inicial/moderada e periodontite avançada, em relação aos sadios.

Observou-se, entre aqueles classificados pelo CPI como com periodontite (inicial/moderada ou avançada), maior porcentagem de indivíduos do sexo masculino $(\mathrm{p}=0,000)$ e de fumantes ( $\mathrm{p}=0,034)$ quando comparados aos demais. Não foram notadas associações estatisticamente significantes entre a condição de saúde bucal (pe- 
Tabela 2

Valores médios (desvios padrão) de variáveis demográficas, antropométricas e bioquímicas segundo a condição de saúde bucal definida pelo índice periodontal comunitário de nipo-brasileiros.

\begin{tabular}{|c|c|c|c|c|c|c|c|c|c|c|}
\hline \multirow[t]{2}{*}{ Variável } & \multicolumn{2}{|c|}{$\begin{array}{l}\text { Sadios } \\
(n=215)\end{array}$} & \multicolumn{2}{|c|}{$\begin{array}{l}\text { Gengivite } \\
(n=513)\end{array}$} & \multicolumn{2}{|c|}{$\begin{array}{c}\text { Periodontite } \\
\text { inicial ou moderada } \\
\qquad(n=85)\end{array}$} & \multicolumn{2}{|c|}{$\begin{array}{l}\text { Periodontite } \\
\text { avançada } \\
(n=18)\end{array}$} & \multicolumn{2}{|c|}{$\begin{array}{l}\text { Edentulismo } \\
\qquad(n=484)\end{array}$} \\
\hline & Média & $d p$ & Média & $d p$ & Média & $d p$ & Média & $\mathrm{dp}$ & Média & $\mathrm{dp}$ \\
\hline Idade (anos) & 52,3 & 11,6 & 52,6 & 10,5 & 52,5 & 11,1 & 58,7 & 10,0 & 65,5 & 9,9 \\
\hline Homa-r * & 2,7 & 2,9 & 2,8 & 2,2 & 2,6 & 3,1 & 2,2 & 2,41 & 2,7 & 3,1 \\
\hline Proteína C-reativa (mg/dl) & 0,2 & 0,4 & 0,2 & 0,4 & 0,5 & 1,0 & 0,2 & 0,3 & 0,3 & 0,6 \\
\hline Leptina (ng/ml) & 7,3 & 6,7 & 7,3 & 6,5 & 6,0 & 6,7 & 5,1 & 3,8 & 7,3 & 6,9 \\
\hline Homocisteína $(\mu \mathrm{m} / \mathrm{l})$ & 11,3 & 8,3 & 10,9 & 5,5 & 13,0 & 15,9 & 13,8 & 9,2 & 11,6 & 4,8 \\
\hline Índice de massa corporal (kg/m²) & 24,5 & 3,8 & 25,2 & 3,8 & 25,1 & 4,5 & 25,5 & 3,9 & 24,8 & 3,9 \\
\hline LDL colesterol ** (mg/dl) & 130,1 & 38,2 & 129,0 & 37,0 & 128,0 & 40,3 & 121,1 & 32,7 & 132,6 & 38,7 \\
\hline Colesterol total (mg/dl) & 212,0 & 43,6 & 213,9 & 41,0 & 211,5 & 45,7 & 201,4 & 34,1 & 217,8 & 42,8 \\
\hline Ácido úrico (mg/dl) & 5,8 & 1,8 & 6,4 & 1,9 & 6,2 & 1,6 & 6,7 & 1,5 & 6,1 & 1,7 \\
\hline Creatinina (mg/dl) & 0,8 & 0,4 & 0,8 & 0,3 & 0,9 & 0,3 & 0,9 & 0,3 & 0,8 & 0,3 \\
\hline
\end{tabular}

* Resistência à insulina avaliada pelo HOMA;

** Lipoproteína de baixa densidade.

riodontite $v s$. sadios) e a geração, escolaridade, presença de síndrome metabólica e hábito de consumir bebidas alcoólicas (dados não apresentados).

Nas Tabelas 3 e 4 apresentam-se os valores dos OR e seus respectivos intervalos de confiança para as variáveis incluídas no estudo segundo a presença da síndrome metabólica - considerando os 318 indivíduos com saúde periodontal ou com qualquer grau de periodontite. Não foi observada associação estatisticamente significante entre as periodontites e a síndrome metabólica $[\mathrm{OR}=1,11(0,67-1,83)]$. As condições que se associaram à síndrome metabólica foram a idade $[\mathrm{OR}=1,02(1,01-1,03)]$, resistência à insulina [OR $=1,58(1,31-1,90)]$, creatinina $[\mathrm{OR}=2,67(1,18$ $6,00)]$, ácido úrico [OR = 1,70 $(1,44-2,01)]$ e IMC elevado [OR = 1,40 (1,26-1,49)].

Na Tabela 5 são apresentados os valores dos OR para o modelo inicial e final que relacionam a síndrome metabólica e a condição periodontal (doente $v s$. sadio). Independentemente de outros fatores de risco, a presença da síndrome metabólica associou-se positivamente aos altos níveis de ácido úrico $[\mathrm{OR}=1,75(1,19-2,57)]$ e ao IMC elevado $[\mathrm{OR}=1,24(1,05-1,48)]$. Assim como foi observado na análise bruta, não foi verificada associação estatisticamente significante entre a síndrome metabólica e as periodontites.

\section{Discussão}

Esse estudo foi planejado com o intuito de estudar a relação entre doenças periodontais e síndrome metabólica na população nipo-brasileira - reconhecidamente afetada por distúrbios metabólicos como o diabetes mellitus e suas complicações. Os principais achados foram a elevada prevalência de doenças periodontais, edentulismo e síndrome metabólica na população investigada e a ausência de associação entre periodontites e a síndrome metabólica. Dos indivíduos investigados, $47 \%$ apresentaram ou gengivite ou periodontite, valor esse considerado elevado, mas compatível com os observados em outros estudos 42,43 , contudo essa comparação deve ser feita com cautela, pois diferentes procedimentos foram utilizados para o diagnóstico e classificação das doenças periodontais.

É referido na literatura que, para qualquer população, a prevalência de periodontites em estágio avançado varia entre 5 a $15 \%$, enquanto que as periodontites em estágio inicial a moderado afetam uma porcentagem bem maior da população 25 . Entretanto, essas estimativas variam muito entre os estudos devido ao emprego de várias definições de "caso de periodontites". Neste estudo, quando se detém especificamente aos casos de periodontites, o número de indivíduos doentes diminui consideravelmente (de $47 \%$ para menos de $10 \%$ com periodontites); esses valores concordam com a baixa prevalência de periodontites 
Número e percentual de indivíduos (porcentagem) e valores dos odds ratios (intervalo com 95\% de confiança) para variáveis demográficas, segundo a presença ou ausência da síndrome metabólica no grupo de indivíduos sadios ou com periodontites $(n=318)$.

\begin{tabular}{|c|c|c|c|c|c|c|c|}
\hline \multirow[t]{2}{*}{ Variável } & \multicolumn{2}{|c|}{ Sim } & \multicolumn{2}{|c|}{ Não } & \multicolumn{2}{|c|}{ Total } & \multirow[t]{2}{*}{ OR (IC95\%) } \\
\hline & $\mathbf{n}$ & $\%$ & $\mathbf{n}$ & $\%$ & $\mathrm{n}$ & $\%$ & \\
\hline CPI & & & & & & & $1,11(0,67-1,83)$ \\
\hline Sadios * & 105 & 48,8 & 110 & 51,2 & 215 & 100,0 & \\
\hline Periodontite ** & 53 & 51,5 & 50 & 48,5 & 103 & 100,0 & \\
\hline Sexo & & & & & & & $1,16(0,93-1,45)$ \\
\hline Masculino & 88 & 56,1 & 69 & 43,9 & 157 & 100,0 & \\
\hline Feminino & 77 & 44,0 & 98 & 56,0 & 175 & 100,0 & \\
\hline Geração & & & & & & & $1,34(0,68-2,71)$ \\
\hline Primeira & 19 & 43,2 & 25 & 56,8 & 44 & 100,0 & \\
\hline Segunda & 138 & 50,5 & 135 & 49,4 & 273 & 100,0 & \\
\hline Tabagismo & & & & & & & $0,91(0,55-1,50)$ \\
\hline Nunca fumou & 109 & 50,5 & 107 & 49,5 & 216 & 100,0 & \\
\hline Fuma/Fumou & 48 & 48,0 & 52 & 52,0 & 100 & 100,0 & \\
\hline Consumo de álcool & & & & & & & $0,87(0,54-1,38)$ \\
\hline Não consome & 95 & 51,1 & 91 & 48,9 & 186 & 100,0 & \\
\hline Consome & 62 & 47,5 & 69 & 52,7 & 131 & 100,0 & \\
\hline Escolaridade (anos) & & & & & & & $0,95(0,42-2,10)$ \\
\hline$<4$ & 65 & 50,0 & 65 & 50,0 & 130 & 100,0 & \\
\hline$\geq 4$ & 18 & 48,7 & 19 & 51,4 & 37 & 100,0 & \\
\hline
\end{tabular}

* Sem sinais clínicos de doenças periodontais nos seis sextantes da boca;

** Com bolsas periodontais $>4 \mathrm{~mm}$ de profundidade, em pelo menos um sextante da boca.

Tabela 4

Médias (desvios padrão) e valores dos odds ratios (intervalo com 95\% de confiança) para variáveis antropométricas e bioquímicas, segundo a presença ou ausência da síndrome metabólica em indivíduos sadios ou com periodontites $(n=318$ ).

\begin{tabular}{|c|c|c|c|c|c|}
\hline \multirow[t]{2}{*}{ Variável } & \multicolumn{2}{|c|}{ Sim } & \multicolumn{2}{|c|}{ Não } & \multirow[t]{2}{*}{ OR (IC95\%) } \\
\hline & $\mathbf{n}$ & $\%$ & $\mathbf{n}$ & $\%$ & \\
\hline Idade (anos) & 55,0 & 10,7 & 50,5 & 12,2 & $1,03(1,01-1,05)$ \\
\hline Homa-r * & 3,5 & 3,7 & 1,8 & 1,4 & $1,58(1,31-1,90)$ \\
\hline Proteína C-reativa (mg/dL) & 0,3 & 0,4 & 0,2 & 0,7 & $1,01(0,73-1,65)$ \\
\hline Leptina (ng/dL) & 7,0 & 6,8 & 6,7 & 6,2 & $1,00(0,97-1,04)$ \\
\hline Homocisteína $(\mu \mathrm{m} / \mathrm{L})$ & 12,5 & 11,8 & 11,1 & 9,4 & $1,01(0,99-1,04)$ \\
\hline Índice de massa corporal $\left(\mathrm{kg} / \mathrm{m}^{2}\right)$ & 26,5 & 4,2 & 22,9 & 2,8 & $1,40(1,26-1,49)$ \\
\hline LDL-colesterol ${ }^{* \star}(\mathrm{mg} / \mathrm{dL})$ & 129,8 & 40,3 & 128,3 & 36,7 & $1,00(0,99-1,00)$ \\
\hline Colesterol total (mg/dL) & 215,8 & 45,6 & 206,8 & 41,3 & $1,00(0,99-1,01)$ \\
\hline Creatinina (mg/dL) & 0,8 & 0,2 & 0,9 & 0,4 & $2,66(1,18-6,00)$ \\
\hline Ácido úrico (mg/dL) & 6,6 & 1,8 & 5,3 & 1,4 & $1,70(1,44-2,01)$ \\
\hline
\end{tabular}

* Resistência à insulina avaliada pelo índice HOMA (resistentes: HOMA-r $\geq 2,4$ );

** Lipoproteína de baixa densidade. 
Valores dos odds ratios (intervalo com 95\% de confiança) para a presença de síndrome metabólica e periodontites ajustados às demais variáveis.

\begin{tabular}{llc}
\hline Variável & \multicolumn{1}{c}{$\begin{array}{c}\text { Síndrome metabólica (sim vs. não) } \\
\text { Modelo final } \\
\text { OR (IC95\%) }\end{array}$} & $\begin{array}{c}\text { Modelo inicial } \\
\text { OR (IC95\%) }\end{array}$ \\
\hline CPI (periodontite vs. higidez) & $0,45(0,14-1,43)$ & $0,44(0,16-1,21)$ \\
Idade (anos) & $0,99(0,94-1,03)$ & - \\
Sexo (masculino vs. feminino) & $0,49(0,14-1,79)$ & $1,50(0,49-4,60)$ \\
Escolaridade $(\geq 4$ anos vs. $<4$ anos) & $1,61(0,44-5,92)$ & - \\
Creatinina (mg/dl) & $1,41(0,32-6,31)$ & $1,75(1,19-2,57)$ \\
Ácido úrico $(\mathrm{mg} / \mathrm{dl})$ & $1,96(1,22-3,13)$ & - \\
Leptina $(\mathrm{ng} / \mathrm{dl})$ & $1,06(0,62-1,82)$ & $0,85(0,33-2,21)$ \\
Homocisteína $(\mu \mathrm{m} / \mathrm{l})$ & $1,05(0,38-2,95)$ & $1,57(0,97-2,55)$ \\
Proteína C-reativa $(\mathrm{mg} / \mathrm{dl})$ & $1,64(0,93-2,89)$ & $1,25(1,05-1,48)$ \\
Índice de massa corporal $\left(\mathrm{kg} / \mathrm{m}^{2}\right)$ & $1,26(1,05-1,52)$ & \\
\hline
\end{tabular}

encontrada na população adulta brasileira - entre 9,9 e 6,3\%, segundo a faixa etária analisada 44 . Uma possível explicação para os baixos valores de prevalência das periodontites na população brasileira adulta (de origem nipônica ou não) é a alta prevalência de edentulismo observada entre brasileiros adultos.

O aumento da prevalência e do agravamento da perda de inserção periodontal com a evolução das periodontites associa-se diretamente ao aumento da idade 45 , porém acredita-se que a maior destruição periodontal nos idosos seja mais reflexo do histórico de doenças periodontais acumuladas ao longo dos anos do que uma condição específica da idade.Concordante com a literatura, neste estudo, indivíduos que apresentaram periodontite avançada ou edentulismo total tinham idade média maior que os sadios ou com gengivite ou com periodontite inicial a moderada. Foi também encontrada uma maior prevalência de periodontites no grupo dos homens. As razões para as diferenças de prevalência entre os sexos não estão bem esclarecidas, mas a literatura sugere que exista, entre os homens, menor atenção à saúde bucal que entre as mulheres 45 .

A maior prevalência de periodontites entre os indivíduos fumantes ou ex-fumantes, quando comparados aos sem esse hábito, também é descrita na literatura 45,46. O tabagismo tem sido considerado um fator de risco para o desenvolvimento das doenças periodontais mesmo em pacientes com bom nível de higiene oral 47,48 , além disso, o controle da doença nos pacientes que fumam é, em geral, mais difícil. Maior gravidade da doença também está relacionada com o hábito de fumar, sendo que o grau de perda óssea alveolar e o número de dentes perdidos são maiores entre os indivíduos fumantes quando comparados aos não fumantes; os mecanismos que explicariam essa relação incluiriam a diminuição da vascularização periodontal, o enfraquecimento da resposta inflamatória e salivação - importantes defesas contra os periodontopatógenos - e o comprometimento de células envolvidas no processo de reparação dos tecidos periodontais.

Neste estudo, o edentulismo foi um achado freqüente $(36,8 \%)$. Para a população brasileira total, essa porcentagem é também elevada. No estudo realizado pelo Ministério da Saúde entre os anos de 2002 e 2003, 11,4\% dos indivíduos com idade entre 35-44 anos foram considerados como tendo edentulismo, já para os com idade entre 65-74 anos, a prevalência de edentulismo foi de $60,8 \% 44$.

A perda dental priva o indivíduo de ingerir determinados alimentos, o que pode comprometer seu estado nutricional e, conseqüentemente, sua saúde geral 49 . Diversos fatores podem estar relacionados ao edentulismo (idade, fumo, estado sócio-econômico, práticas de higiene inadequadas, atitudes sociais e culturais, bem como a filosofia do profissional dentista). Sabe-se que as periodontites são consideradas um dos mais representativos fatores de risco para a perda precoce dos dentes na população adulta. Segundo Nunn 50, a medida mais acurada para verificar a influência de um fator de risco na progressão das periodontites é a perda dental. Assim, a elevada freqüência de edentulismo entre os nipo- 
brasileiros pode sugerir um histórico prévio de periodontites.

As perdas de inserção foram altas também nessa população (cerca de $50 \%$ dos indivíduos examinados). De acordo com Nunn ${ }^{50}$, a perda clínica de inserção periodontal está fortemente associada às perdas dentais por doenças periodontais, o que ratifica a possibilidade de um histórico prévio de periodontites pelos nipo-brasileiros com edentulismo. Contudo, a ausência de informações sobre medidas de inserção clínica antes da presença das periodontites não permite afirmar que a perda de inserção foi devida exclusivamente às doenças periodontais, pois outros fatores como má oclusão e maus hábitos poderiam também ter contribuído para a elevada perda clínica de inserção periodontal 51 .

Como era esperado, nesse estudo, indivíduos com síndrome metabólica se caracterizaram por pior perfil antropométrico e metabólico, destacando-se o número elevado de nipo-brasileiros com sobrepeso, resistência à insulina, níveis séricos elevados de creatinina e ácido úrico.

As alterações metabólicas que compõem a síndrome metabólica (intolerância à glicose, obesidade, hipertensão arterial, dislipidemias) são alvos freqüentes de estudo e intervenção de órgãos internacionais e nacionais de saúde pela alta mortalidade e morbidade que suscitam. Conhecer os possíveis fatores de risco dessas doenças para preveni-las ou tratá-las é prioritário; as evidências existentes indicam que condições inflamatórias podem contribuir para o aparecimento desses distúrbios. Microorganismos periodontopatogênicos produzem endotoxinas na forma de lipopolissacarídeos que são ativadores da resposta imune destrutiva dos tecidos do hospedeiro. Sabe-se que a destruição dos tecidos periodontais é mediada por citocinas pró-inflamatórias (IL-1, IL-6 e IL-8, PGE 2 , TNF- $\alpha$ ). Essas cito- cinas estão suficientemente elevadas no fluído crevicular gengival para ser lançadas na corrente sanguínea, sendo detectáveis em ensaios séricos (1 a 3 micro mol/l); assim, pode-se supor que, em alguma medida, essas substâncias possam contribuir para o desenvolvimento da síndrome metabólica e doenças associadas 27,28,52,53.

Nos últimos anos, diversas pesquisas relacionando saúde bucal e saúde sistêmica foram realizadas no intuito de atingir a meta proposta pela OMS para o ano de 2020 de minimizar o impacto das doenças de origem oral e craniofacial sobre a saúde e o desenvolvimento psicossocial 54. Em especial, foi considerada a possibilidade de que as doenças periodontais fossem fatores de risco para o desenvolvimento ou agravamento de condições sistêmicas como a resistência à insulina, o diabetes mellitus do tipo 2, a obesidade e as dislipidemias 55,56,57,58,59,60,61,62,63,64; apesar de o delineamento transversal deste estudo limitar o estabelecimento de relação causa-efeito entre a doenças periodontais e a síndrome metabólica, a ausência de associação observada entre essas variáveis sugere que, pelo menos entre esses nipo-brasileiros, outros fatores seriam responsáveis pelo aparecimento da síndrome metabólica. Contudo, deve-se considerar que a baixa prevalência de periodontites observada pode não ter permitido detectar essa associação - se de fato ela existir.

Em síntese, na população nipo-brasileira, a prevalência de alterações periodontais clínicas foi elevada. Entre os pacientes com periodontite, houve uma discreta elevação na prevalência da síndrome metabólica, quando comparados ao grupo dos que não tinham periodontite. Porém essa associação não pode ser comprovada estatisticamente. Apesar da ausência de associação, não pode ser descartada a possibilidade de que essa relação exista. 


\section{Resumo}

Verificou-se, num estudo transversal, a relação entre as doenças periodontais e a síndrome metabólica. Foram analisados dados de 1.315 nipo-brasileiros com idade entre 30 e 92 anos. Os indivíduos foram submetidos a exames físicos, odontológicos e laboratoriais. Os dados foram descritos através de médias e porcentagens e a associação entre as variáveis foi verificada por meio de regressão logística. Do total de sujeitos examinados, 215 (16,4\%) apresentaram higidez periodontal, 484 (36,8\%) edentulismo total, 513 (39\%) gengivites, $85(6,5 \%)$ periodontite inicial ou moderada e 18 (1,4\%) periodontite avançada. A prevalência de síndrome metabólica entre os nipo-brasileiros foi 54,3\%, e apesar de esta ter sido maior entre os que apresentaram periodontites quando comparados aos sem nenhum comprometimento da saúde bucal (51,5\% vs. 48,8\%) essa associação não foi estatisticamente significante. Neste estudo os indivíduos com síndrome metabólica se caracterizaram por pior perfil antropométrico e metabólico.

Síndrome X Metabólica; Diabetes Mellitus; Dislipidemias; Doenças Periodontais; Obesidade

\section{Referências}

1. World Health Organization. Definition, diagnosis and classification of diabetes mellitus and its complications. Geneva: World Health Organization; 1999.

2. National Cholesterol Education Program. Expert panel on detection, evaluation, and treatment of high blood cholesterol in adults (Adults Treatment Panel III). JAMA 2001; 285:2486-97.

3. Grundy SM, Brewer Jr. HB, Cleeman JI, Smith Jr. SC, Lenfant C; American Heart Association; National Heart, Lung, and Blood Institute. Definition of metabolic syndrome: report of the National Heart, Lung, and Blood Institute/American Heart Association conference on scientific issues related to definition. Circulation 2004; 109:433-8.

4. Reaven GM. Role of insulin resistance in human disease. Diabetes 1988; 37:1595-607.

5. Reaven GM. Role of insulin resistance in human disease (syndrome $\mathrm{X}$ ): an expanded definition. Annu Rev Med 1993; 44:121-31.

6. Lakka HM, Laaksonen DE, Lakka TA, Niskanen LK, Kumpusalo E, Tuomilehto J, et al. The metabolic syndrome and total and cardiovascular disease mortality in middle-aged men. JAMA 2002; 288:2709-16.

\section{Colaboradores}

P. K. O. Borges participou do planejamento do estudo, análise estatística dos dados e elaboração do manuscrito. S. G. A. Gimeno contribuiu no planejamento do estudo, coleta de dados, análise estatística dos dados e revisão do manuscrito. N. E. Tomita participou do planejamento do estudo e da coleta de dados. S. R. Ferreira contribuiu no planejamento do estudo e na coleta de dados.

\section{Outros membros do Japanese-Brazilian Diabetes Study Group}

N. Barros Jr., M. A. Cardoso, R. Chaim, V. D’Almeida L. J. Franco, H. Harima, A. Hirai, A. T. Hirai, M. Kikuchi, L. Matsumura, R. S. Moisés, K. Osiro, K. Wakisaka.
7. Rosenbaum P, Gimeno SGA, Sanudo A, Franco LJ, Ferreira SRG. Analysis of criteria for metabolic syndrome in a population-based study of Japanese-Brazilians 2005. Diabetes Obes Metab 2005; 7:352-9.

8. International Diabetes Federation/World Health Organization. The IDF consensus worldwide definition of the metabolic syndrome, 2005. http:// www.idf.org/webcast/pdf/IDF_backgrounder_ 1.pdf (acessado em 10/Mai/2005).

9. Laaksonen DE, Lakka HM, Niskanen LK, Kaplan GA, Salonen JT, Lakka TA. Metabolic syndrome and development of diabetes mellitus: application and validation of recently suggested definitions of metabolic syndrome in a prospective cohort study. Am J Epidemiol 2002; 156:1070-7.

10. Ford ES, Giles WH, Dietz WH. Prevalence of the metabolic syndrome among US adults: findings from the third National Health and Nutrition Examination Survey. JAMA 2002; 287:356-9.

11. Meigs JB, Wilson PWF, Nathan DM, D'Agostino RB, Williams K, Haffner SM. Prevalence and characteristics of the metabolic syndrome in the San Antonio Heart and Framingham Offspring Studies. Diabetes 2003; 52:2160-7. 
12. Shimamoto K. Epidemiologic study on metabolic syndrome - comparison between Japan and western countries. Nippon Rinsho 2004; 62:1053-8.

13. Sone H, Mizuno S, Fujii H, Yoshimura Y, Yamasaki Y, Ishibashi S, et al. Is the diagnosis of metabolic syndrome useful for predicting cardiovascular disease in Asian diabetic patients? Diabetes Care 2005; 28:1463-71.

14. Lerario DG, Gimeno SGA, Franco LJ, Iunes M, Ferreira SRG. Prevalence of weight excess and implications of abdominal fat distribution for the metabolic syndrome in Japanese-Brazilians. Rev Saúde Pública 2002; 36:4-11.

15. Gimeno SGA, Ferreira SRG, Franco LJ, Hirai AT, Matsumura L, Moisés RS. Prevalence and 7-year incidence of Type II diabetes mellitus in a Japanese-Brazilian population: an alarming public health problem. Diabetologia 2002; 45:1635-8.

16. Esposito K, Marfella R, Ciotola M, Palo C, Giugliano F, Giugliano G, et al. Effect of a Mediterranean-style diet on endothelial dysfunction and markers of vascular inflammation in the metabolic syndrome: a randomized trial. JAMA 2004; 292: 1440-6.

17. Elkind MSH, Rundek T, Sciacca RR, Ramas R, Chen HJ, Boden-Abdala B, et al. Interleukin-2 levels are associated with carotid artery intima-media thickness. Atherosclerosis 2005; 180:181-7.

18. Erdmann E. Diabetes and cardiovascular risk markers. Curr Med Res Opin 2005; 21:521-8.

19. Fantuzzi G. Adipose tissue, adipokines, and inflamation. J Allergy Clin Immunol 2005; 115:911-9.

20. American Academy of Periodontology. The pathogenesis of periodontal disease. J Periodontol 1999; 70:457-70.

21. Carranza Jr. FA, Newman MG. Periodontia clínica. 8a Ed. Rio de Janeiro: Editora Guanabara Koogan; 1997.

22. Lindhe J. Tratado de periodontia clínica e implantologia oral. Rio de Janeiro: Editora Guanabara Koogan; 1992.

23. Anonymous. Consensus report. Periodontal disease: epidemiology and diagnosis. Ann Periodontol 1996; 1:216-22.

24. American Academy of Periodontology. Periodontal disease as a potential risk factor for systemic disease. J Periodontol 1998; 69:841-50.

25. Albandar JM, Rams TE. Global epidemiology of periodontal disease: an overview. Periodontol 2000 2002; 29:7-10.

26. Kigman A, Albandar JM. Methodological aspects of epidemiological studies of periodontal disease. Periodontol 2000 2002; 29:11-30.

27. Clarkson JJ, McLoughlin J. Role of fluoride in oral health promotion. Int Dent J 2000; 50:119-28.

28. Page RC. The pathobiology of periodontal diseases may affect systemic disease: inversion of a paradigm. Ann Periodontol 1998, 3:108-20.

29. Nishimura F, Murayama Y. Periodontal inflammation and insulin resistance - lessons from obesity. J Dent Res 2001; 80:1690-4.

30. Uysal KT, Wiesbrock SM, Marino MW, Hotamisgil GS. Protection from obesity-induced insulin resistance in mice lacking TNF-alfa function. Nature 1997; 389:610-4.
31. Ferreira SR, Iunes M, Franco LJ, Iochida LC, Hirai A, Vivolo MA. Disturbances of glucose and lipid metabolism in first and second generation Japanese-Brazilians. Japanese-Brazilian Diabetes Study Group. Diabetes Res Clin Pract 1996; 34 Suppl: S59-63.

32. Tomita NE, Chinellato LE, Pernambuco RA, Lauris JR, Franco LJ; Grupo de Estudo Diabetes em Nipo-Brasileiros. Condições periodontais e diabetes mellitus na população nipo-brasileira. Rev Saúde Pública 2002; 36:607-13.

33. Pernambuco RA. Diabetes mellitus e condições periodontais na população nipo-brasileira de Bauru-SP-Brasil [Dissertação de Mestrado]. Bauru: Faculdade de Odontologia, Universidade de São Paulo; 2001.

34. World Health Organization/International Obesity Task Force. The Asia-Pacific perspective: redefining obesity and its treatment, 2000. http://www. wpro.who.int/NR/rdonlyres/B924BFA6-A06143AE-8DCA-0AE82A8F66D2/0/obesityinthepacific.pdf (acessado em 15/Out/2005).

35. Matthews DR, Hosker JP, Rudenski AS, Naylor BA, Treacher DF, Turner RC. Homeostasis model assessment insulin resistance and beta-cell function from fasting plasma glucose and insulin concentrations in man. Diabetologia 1985, 28:412-9.

36. Pfeiffer CM, Huff DL, Gunter EW. Rapid and accurate HPLC assay for plasma total homocysteine and cysteine in a clinical laboratory setting. Clin Chem 1999; 45:290-2.

37. World Health Organization. Oral health surveys - basic methods. Geneva: World Health Organization; 1997.

38. Pinto VG. Saúde bucal coletiva. 4ạ Ed. São Paulo: Editora Santos; 2000.

39. Anonymous. Parameter on plaque-induced gingivitis. American Academy of Periodontology. J Periodontol 2003; 71 (5 Suppl):851-2.

40. Hosmer JR, Lemeshow S. Applied logistic regression. New York: John Wiley \& Sons; 1989.

41. World Medical Association. Declaration of Helsinki: ethical principles for medical research involving human subjects. http://www.wma.net/e/ policy/b3.htm (acessado em 27/Nov/2005).

42. Oppermann R, Rosing CK, Gjermo P, Susin C. Periodontal disease in Central and South America. Periodontol 2000 2002; 29:70-8.

43. Miyazaki H, Hanada N, Andoh MI, Yamashita Y, Saito T, Sogame A, et al. Periodontal disease prevalence in different age groups in Japan, as assessed according to the CPITN. Community Dent Oral Epidemiol 1989; 17:71-4.

44. Ministério da Saúde. Condições de saúde bucal na população brasileira 2002-2003. Brasília: Ministério da Saúde; 2005.

45. Burt B; Research, Science and Therapy Committee of the American Academy of Periodontology. Position paper: epidemiology of periodontal disease. J Periodontol 2005; 76:1406-19.

46. Hidalgo FR. Smoking and periodontal disease. Periodontol 2000 2003; 32:50-8.

47. Tomar SL, Asma S. Smoking-attributable periodontitis in the United States: findings from NHANES III. J Periodontol 2000; 71:743-51. 
48. Bergstrom J, Eliasson S, Dock J. Tobacco use as risk factor. J Periodontol 1994; 65 Suppl:545-50.

49. Shah N, Parkash H, Sunderam KR. Edentulousness, denture wear and denture needs of Indian elderly - a community-based study. J Oral Rehabil 2004; 31:467-76

50. Nunn ME. Understanding the etiology of periodontitis: an overview of periodontal risk factors. Periodontol 2000 2003; 32:11-23.

51. Page RC, Sturdivant EC. Non-inflammatory destructive periodontal disease (NDPD). Periodontol 2000 2002; 30:24-39.

52. Ritchie CS, Kinane DF. Nutrition, inflammation, and periodontal disease. Nutrition 2003; 19:475-6.

53. Research, Science and Therapy Committee, American Academy of Periodontology. Periodontal management of patients with cardiovascular disease. J Periodontol 2002; 73:954-68.

54. Hobdell M, Petersen PE, Clarkson J, Johson N. Global goal for oral health 2020. Int Dent J 2003; 53:285-8.

55. Knowler WC, Pettitt DJ, Saad MF, Bennet PH. Diabetes mellitus in Pima Indians: incidence, risk factors and pathogenesis. Diabetes Metab Rev 1990; 6:1-27.

56. Grossi SG, Skrepcinski FB, DeCaro T, Zambon JJ, Cummins D, Genco RJ. Response to periodonta therapy in diabetics and smokers. J Periodontol 1996; 67:1094-102.

57. Taylor GW, Burt BA, Becker MP, Genco RJ, Shlossman M, Knowler WC, et al. Severe periodontitis and risk for poor glycemic control in patients with non-insulin-dependent diabetes mellitus. J Periodontol 1996; 67:1085-93.
58. DeStefano F, Anda RF, Kahn HS, Williamson DF, Russel CM. Dental disease and risk of coronary heart disease. BMJ 1993; 306:688-91.

59. Katz J, Chaushu G, Sharabi Y. On the association between hypercholesterolemia, cardiovascular disease and severe periodontal disease. J Clin Periodontol 2001; 28:865-8.

60. Losche W, Karapetow F, Pohl A, Kocher T. Plasma lipid and blood glucose levels in patients with destructive periodontal disease. J Clin Periodontol 2000; 27:537-41.

61. Nishimura F, Murayama Y. Periodontal inflammation and insulin resistance- lessons from obesity. J Dent Res 2001; 80:1690-4.

62. Iwamoto Y, Nishimura F, Nakagawa M, Sugimoto $\mathrm{H}$, Shikata K, Makino H, et al. The effect of antimicrobial periodontal treatment on circulating tumor necrosis factor-alpha and glycated hemoglobin level in patients with type 2 diabetes. J Periodontol 2001; 72:774-8.

63. Nishimura F, Iwamoto Y, Mineshiba J, Shimizu A, Soga Y, Murayama Y. Periodontal disease and diabetes mellitus: the role of tumor necrosis factoralpha in 2-way relationship. J Periodontol 2003; 74:97-102.

64. Nishimura F, Soga Y, Iwamoto Y, Kudo C, Murayama Y. Periodontal disease as part of the insulin resistance syndrome in diabetic patients. J Int Acad Periodontol 2005; 7:16-20.

Recebido em 30/Nov/2005

Versão final reapresentada em 01/Jun/2006 Aprovado em 26/Jul/2006 